\title{
Analysis of log-periodic dipole arrays with boundary elements
}

\author{
D. Poljak ${ }^{1}$, V. Doric ${ }^{1}$, M. Birkic ${ }^{2} \&$ D. Kosor ${ }^{3}$ \\ ${ }^{1}$ Department of Electronics, University of Split, Croatia \\ ${ }^{2}$ Technical Department, Air Traffic Center Pula, Croatia \\ ${ }^{3}$ Technical Department, Air Traffic Center Split, Croatia
}

\begin{abstract}
The paper deals with a frequency domain analysis of a log-periodic dipole array (LPDA) antenna using boundary element analysis. The mathematical model is based on the set of corresponding coupled Pocklington integro-differential equations. Once the current distribution along the wires is obtaining, it is possible to determine the radiation characteristics of LPDA. The set of coupled Pocklington integro-differential equations is handled via the Galerkin-Bubnov scheme of the Indirect Boundary Element Method (GB-IBEM). The field irradiated by LPDA is also assessed using BEM formalism.

Keywords: log-periodic dipole arrays, set of Pocklington equations, boundary elements, frequency domain analysis.
\end{abstract}

\section{Introduction}

A log-pariodic antenna is a radiating system having a structure providing that its impedance and radiation properties repeat periodically as the logarithm of frequency $[1,2]$. As the variations over a wide frequency range of interest are negligible, log-periodic antennas are usually considered to be frequencyindependent.These antennas operate in the VHF and UHF bands thus covering the frequency range from $30 \mathrm{MHz}$ to $3 \mathrm{GHz}$.

The log-periodic dipole array (LPDA) antenna is a series-fed array of parallel straight wires having successively increasing lengths outward from the feeding point at the apex. There are smaller elements in front of and larger elements behind each dipole thus producing a directional primary pattern in the same way as a Yagi antenna. Contrary to the Yagi-Uda array where only one element of the array is directly fed, while the other wires operate in a parasitic mode, all the elements of the LPDA are connected to the feeder. 
Log-periodic dipole array antennas are driven by attaching dipole arms to two parallel conductors which represent a transmission line [1]. The transmission line feeder is crossed between each dipole thus reversing the direction of fire. The LPDA antennas are found to be easy to optimize, while the crossing of the feeder between each dipole element leads to a mutual cancellation of backlobe components from the individual elements yielding to a very low level of backlobe radiation (around $25 \mathrm{~dB}$ below main lobe gain at $\mathrm{HF}$ and $35 \mathrm{~dB}$ at VHF and UHF).

To maintain the geometrical pattern of the LPDA to achieve a trully logperiodic configuration, an infinite configuration would be required. However, the practical broadband radiator configuration is truncated at both ends which limits the frequency of operation to a given bandwidth. The cutoff frequencies of the truncated structure is determined by the electrical lengths of the largest and shortest elements of the structure $[1,2]$. It is worth mentioning that the use of logarithmic antenna arrays is very often related with electronic beam steering. An important application of LPDA antennas is in air traffic. Landing is one of teh most important issues of every successful flight. Although the majority of landings can be conducted solely with visual cues, aircraft must frequently land in weather conditions that require electronic assistance to the pilot or to the autopilot.

Log-periodic dipole antenna is an essential part of localizer antenna arrays. A typical localizer antenna system is a part of the electronic systems known as Instrumental Landing System (ILS). Localizer shapes a radiation pattern in a way to provide lateral guidance to the aircraft beginning its descent, intercepting the projected runway center line, and then making a final approach.

This work deals with a boundary element modeling of LPDA antennas. The formulation in the frequency domain is based on a set of coupled Pocklington integro-differential equations for radiation of multiplethin wires above a lossy ground. The set of Pocklington equations is solved via the Galerkin-Bubnov variant of the indirect Boundary Element Method (GB-IBEM) [4]. Once the induced currents along the LPDA are determined the radiation pattern is assessed using BEM formalism, as well.

\section{Formulation}

Modeling of multiple wire configurations above a lossy ground is an important part in antenna design and electromagnetic compatibility (EMC) studies [2, 5-8]. This section firstly deals with an assessment of the current induced along multiple wires above a lossy ground at different heights. Once the currents along the wire array have been obtained, the equations for related radiated field are presented [8]. In this case, the geometry of interest is LPDA shown in Fig 2. The length of any other wire is obtained by multiplying the previous length and factor $\tau[2]$ :

$$
\tau=\frac{L_{n+1}}{L_{n}}
$$




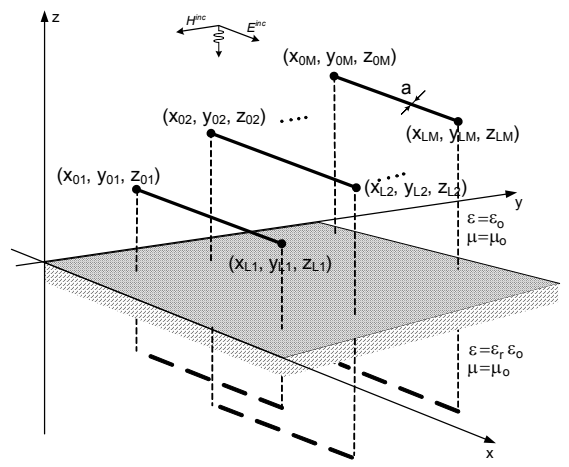

Figure 1: Wires above a lossy ground at different heights.

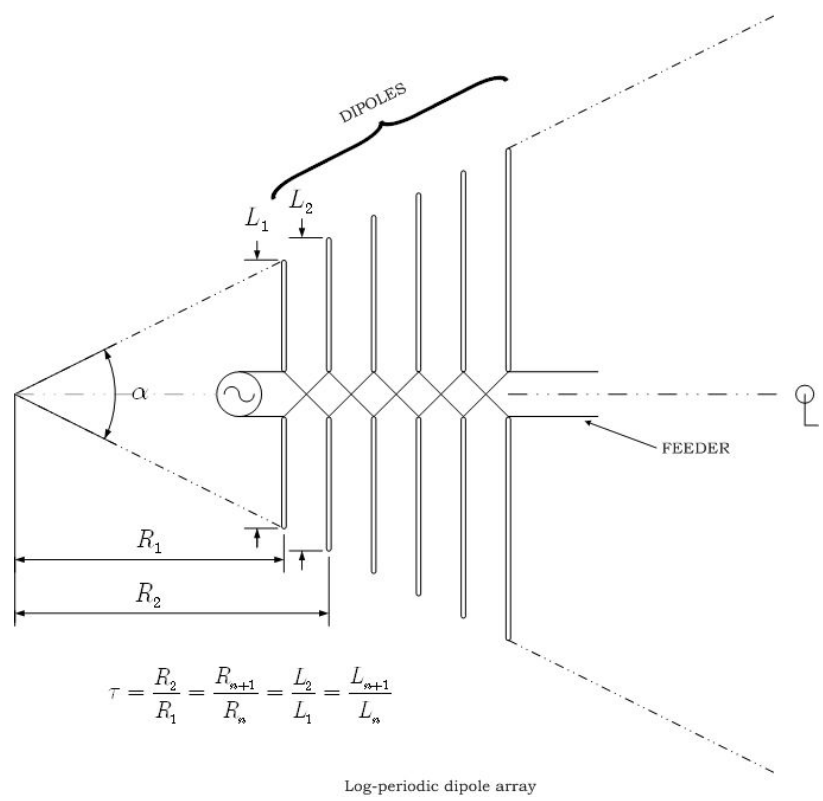

Figure 2: $\quad$ LPDA geometry.

The current distribution along the multiple wire structure shown in Fig 1 is governed by the set of Pocklington integro-differential equations for half-space problems given by [8]:

$$
\begin{aligned}
E_{x}^{e x c} & =-\frac{1}{j 4 \pi \omega \varepsilon_{0}} \sum_{n=1}^{M} \int_{L_{n} / 2}^{L_{n} / 2}\left[\frac{\partial^{2}}{\partial x^{2}}+k_{1}^{2}\right]\left[g_{0 m n}\left(x, x^{\prime}\right)-R_{T M}^{\prime} g_{i m n}\left(x, x^{\prime}\right)\right] I_{n}\left(x^{\prime}\right) d x^{\prime} \\
& m=1,2, \ldots M
\end{aligned}
$$


where $M$ is the total number of wires and $I_{n}\left(x^{\prime}\right)$ is the unknown current distribution induced on the $n$-th wire. Furthermore, $g_{0 m n}\left(x, x^{\prime}\right)$ denotes the free space Green function:

$$
g_{0 m n}\left(x, x^{\prime}\right)=\frac{e^{-j k_{1} R_{1 m n}}}{R_{1 m n}}
$$

while $g_{\text {imn }}\left(x, x^{\prime}\right)$ is, in accordance to the image theory, given by:

$$
g_{\text {imn }}\left(x, x^{\prime}\right)=\frac{e^{-j k_{1} R_{2 m n}}}{R_{2 m n}}
$$

where $k_{l}$ is the propagation constant of free space:

$$
k_{1}^{2}=\omega^{2} \mu_{0} \varepsilon_{0}
$$

and $R_{I m n}$ and $R_{2 m n}$ are distances from the source point and from the corresponding image, respectively to the observation point of interest.

The influence of an imperfectly conducting lower medium is taken into account via the Fresnel plane wave reflection coefficient [RC] [5-8]:

$$
R_{T M}^{\prime}=\frac{\underline{n} \cos \theta^{\prime}-\sqrt{\underline{n}-\sin ^{2} \theta^{\prime}}}{\underline{n} \cos \theta^{\prime}+\sqrt{\underline{n}-\sin ^{2} \theta^{\prime}}}
$$

where $\theta^{\prime}$ and $\underline{n}$ are given by:

$$
\theta^{\prime}=\operatorname{arctg} \frac{\left|x-x^{\prime}\right|}{2 h} \quad m=n \quad \theta^{\prime}=\operatorname{arctg} \frac{R_{1 m n}}{2 h} \quad m \neq n, \underline{n}=\frac{\varepsilon_{\text {eff }}}{\varepsilon_{0}}
$$

and $\varepsilon_{e f f}$ is the complex permittivity of the ground:

$$
\varepsilon_{\text {eff }}=\varepsilon_{r} \varepsilon_{0}-j \frac{\sigma}{\omega}
$$

The electric field (normal incidence) at height $z$ above finitely conducting half-space can be written, as follows:

$$
E^{i n c}=E_{0} e^{-j k_{1}\left(z_{U}-z\right)}
$$

where $E_{0}$ denotes the field amplitude, and $z_{U}$ is the position of the highest antenna among $M$ wires in total: $z_{U}=\max \left(z_{1}, z_{2}, \ldots z, \ldots z_{M}\right)$.

The earth- reflected field component is given by:

$$
E^{r e f}=E_{0} R_{T M} e^{-j k_{1}\left(z_{U}+z\right)}
$$

The total field above ground is composed from both incident and reflected field component:

$$
E^{e x c}=E^{i n c}+E^{r e f}
$$

The electric field components irradiated by the array are given, as follows [8]:

$$
E_{x}=\frac{1}{j 4 \pi \omega \varepsilon_{0}} \sum_{n=1}^{M}\left[-\int_{-L_{n}}^{L_{n}} \frac{\partial I_{n}\left(x^{\prime}\right)}{\partial x^{\prime}} \frac{\partial G_{n m}\left(x, x^{\prime}\right)}{\partial x^{\prime}} d x^{\prime}+k^{2} \int_{-L_{n}}^{L_{n}} G_{n m}\left(x, x^{\prime}\right) I_{n}\left(x^{\prime}\right) d x^{\prime}\right]
$$




$$
\begin{aligned}
& E_{y}=\frac{1}{j 4 \pi \omega \varepsilon_{0}} \sum_{n=1}^{M} \int_{-L_{n}}^{L_{n}} \frac{\partial I_{n}\left(x^{\prime}\right)}{\partial x^{\prime}} \frac{\partial G_{n m}\left(x, x^{\prime}\right)}{\partial y} d x^{\prime} \\
& E_{z}=\frac{1}{j 4 \pi \omega \varepsilon_{0}} \sum_{n=1}^{M} \int_{-L_{n}}^{L_{n}} \frac{\partial I_{n}\left(x^{\prime}\right)}{\partial x^{\prime}} \frac{\partial G_{n m}\left(x, x^{\prime}\right)}{\partial z} d x^{\prime}
\end{aligned}
$$

where the corresponding Green function is given by:

$$
G_{n m}\left(x, x^{\prime}\right)=g_{0 n m}\left(x, x^{\prime}\right)-R_{T M} g_{i n m}\left(x, x^{\prime}\right)
$$

\section{Boundary element procedures}

The Galerkin boundary element procedure for the treatment of the system of integro-differential equations (1), using linear approximation of the solution, starts by applying the standard representation of the unknown current along a segment $[4,8]$ :

$$
I\left(x^{\prime}\right)=I_{1 i} \frac{x_{2 i}-x^{\prime}}{\Delta x}+I_{2 i} \frac{x^{\prime}-x_{1 i}}{\Delta x}
$$

where $I_{l i}$ and $I_{2 i}$ are current values at the local nodes of the $i$-th boundary element, with coordinates $x_{1 i}$ and $x_{2 i}, \Delta x=x_{2 i}-x_{1 i}$ denotes a dimension of the element.

Performing certain mathematical manipulations and the boundary element discretisation of the wires results in the following matrix equation $[4,8]$ :

$$
\sum_{k=1}^{N_{e}}[Z]_{p k}\{I\}_{k}=\{V\}_{p} \quad p=1,2, \ldots, M
$$

where $N_{e}$ is the total number of elements along the actual multiple wire configuration, and $[Z]_{p k}$ is the interaction matrix representing the mutual impedance between each segment on the $i$-th (source) wire to every segment on the $j$-th (observation) wire $[4,8]$ :

$$
[Z]_{p k}^{e}=-\int_{\Delta l_{p}} \int_{\Delta l_{k}}\{D\}_{p}\left\{D^{\prime}\right\}_{k}^{T} g_{j i}\left(x, x^{\prime}\right) d x^{\prime} d x+k^{2} \int_{\Delta l_{p}} \int_{\Delta l_{k}}\{f\}_{l}\left\{f^{\prime}\right\}_{k}^{T} g_{j i}\left(x, x^{\prime}\right) d x^{\prime} d x
$$

Vectors $\{f\}$ and $\left\{f^{\prime}\right\}$ contain shape functions $f_{n}(x)$ and $f_{n}\left(x^{\prime}\right)$, while $\{D\}$ and $\left\{D^{\prime}\right\}$ contain their derivatives. The right side vector $\{V\}_{p}$ represents the voltage along the $p$-th segment and it is given as follows $[4,8]$ :

$$
\{V\}_{p}=-j 4 \pi \omega \varepsilon_{0} \int_{\Delta l_{p}} E_{x}^{i n c}(x)\{f\}_{p} d x
$$

Transmitting mode implies that the vector (19) differs from zero only in the feed-gap area of each wire, Fig 2, and the excitation field can be written, as follows:

$$
E_{x}^{e x c}=\frac{V_{g}}{\Delta l_{g}}
$$

where $V_{g}$ is the feed voltage and $\Delta l_{g}$ is the feed-gap width. 
Once computing the currents along the wires the radiated field can be computed. Applying the BEM formalism to field expressions (12)-(14) it follows:

$$
\begin{gathered}
E_{x}=\frac{1}{j 4 \pi \omega \varepsilon_{0}} \sum_{n=1}^{M} \sum_{i=1}^{N_{j}}\left[-\frac{I_{i+1, n}-I_{i, n}}{\Delta x} \int_{x_{i, n}}^{x_{i+1, n}} \frac{\partial G_{n m}\left(x, x^{\prime}\right)}{\partial x^{\prime}} d x^{\prime}+k^{2} \int_{x_{i, n}}^{x_{i+1, n}} G_{n m}\left(x, x^{\prime}\right) I_{i n}\left(x^{\prime}\right) d x^{\prime}\right] \\
m=1,2, \ldots, M \\
E_{y}=\frac{1}{j 4 \pi \omega \varepsilon_{0}} \sum_{n=1}^{M} \sum_{i=1}^{N_{j}} \frac{I_{i+1, n}-I_{i, n}}{\Delta x} \int_{x_{i, n}}^{x_{i+1, n}} \frac{\partial G_{n m}\left(x, x^{\prime}\right)}{\partial y} d x^{\prime} ; m=1,2, \ldots, M \\
E_{z}=\frac{1}{j 4 \pi \omega \varepsilon_{0}} \sum_{n=1}^{M} \sum_{i=1}^{N_{j}} \frac{I_{i+1, n}-I_{i, n}}{\Delta x} \int_{x_{i, n}}^{x_{i+1, n}} \frac{\partial G_{n m}\left(x, x^{\prime}\right)}{\partial z} d x^{\prime} ; m=1,2, \ldots, M
\end{gathered}
$$

where $N_{j}$ denotes the total number of boundary elements on the $j$-th wire.

The integrals in (21)-(23) are evaluated using Gaussian quadrature. To avoid the problem of the Green function quasi-singularity its first derivative is approximated by means of a central finite difference approximation $[4,8]$.

\section{Computational example}

The computational example is related to the LPDA composed from 12 dipoles insulated in free space. The radius of all wires is $a=0.004 \mathrm{~m}$ while the length of

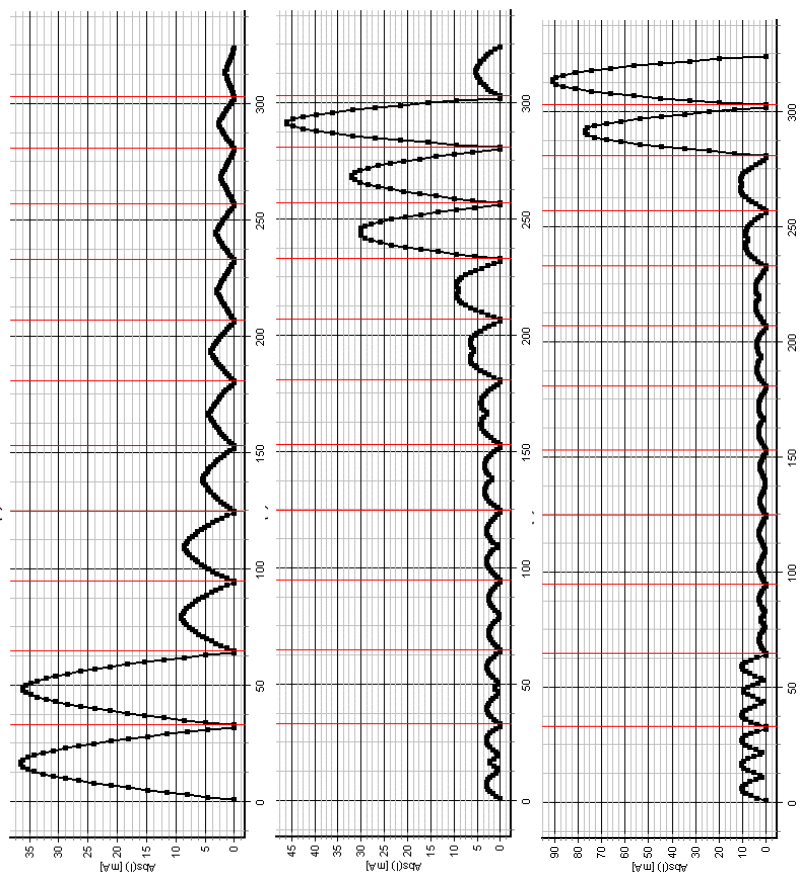

Figure 3: Absolute value of current distribution along 12 dipoles versus BEM nodes at $f=100 \mathrm{MHz}, f=250 \mathrm{MHz}$ and $f=300 \mathrm{MHz}$. 
wires are determined by the length of $1 \mathrm{st}$ wire $L_{l}=1.5 \mathrm{~m}$, and factor $\tau=0.9$. All dipoles are fed by the voltage generator $V_{g}=1 \mathrm{~V}$ with variable phase (each time phase is changed for $180^{\circ}$ ). The operating frequency is varied from $100 \mathrm{MHz}$ to $300 \mathrm{MHz}$. Figure 3 shows the absolute value of currents induced along all 12 dipoles versus BEM nodes at $f=100 \mathrm{MHz}, f=250 \mathrm{MHz}$ and $f=300 \mathrm{MHz}$. Figs 4 to 6 show the related field patterns at $f=100 \mathrm{MHz}, f=250 \mathrm{MHz}$ and $f=300 \mathrm{MHz}$.
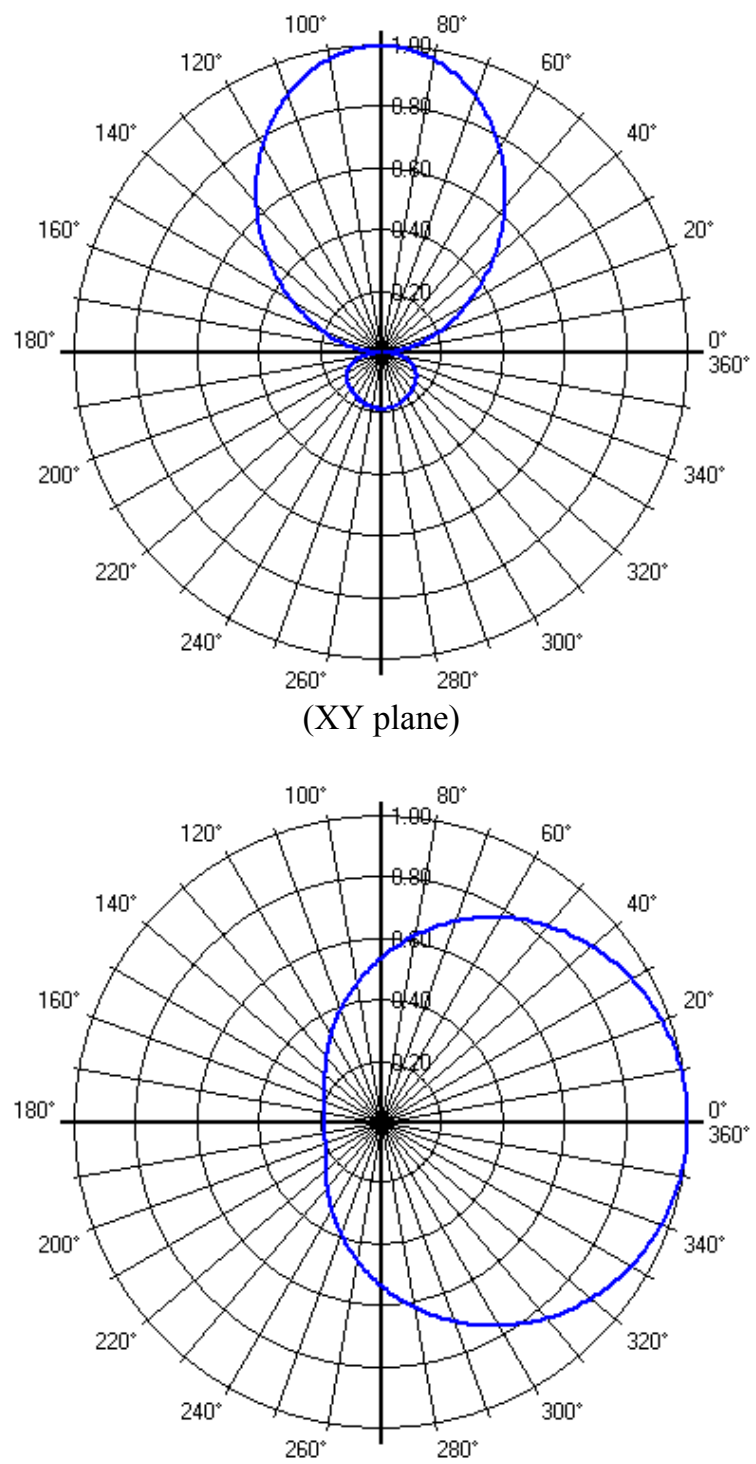

(YZ plane)

Figure 4: $\quad$ Radiation pattern at $f=100 \mathrm{MHz}$. 

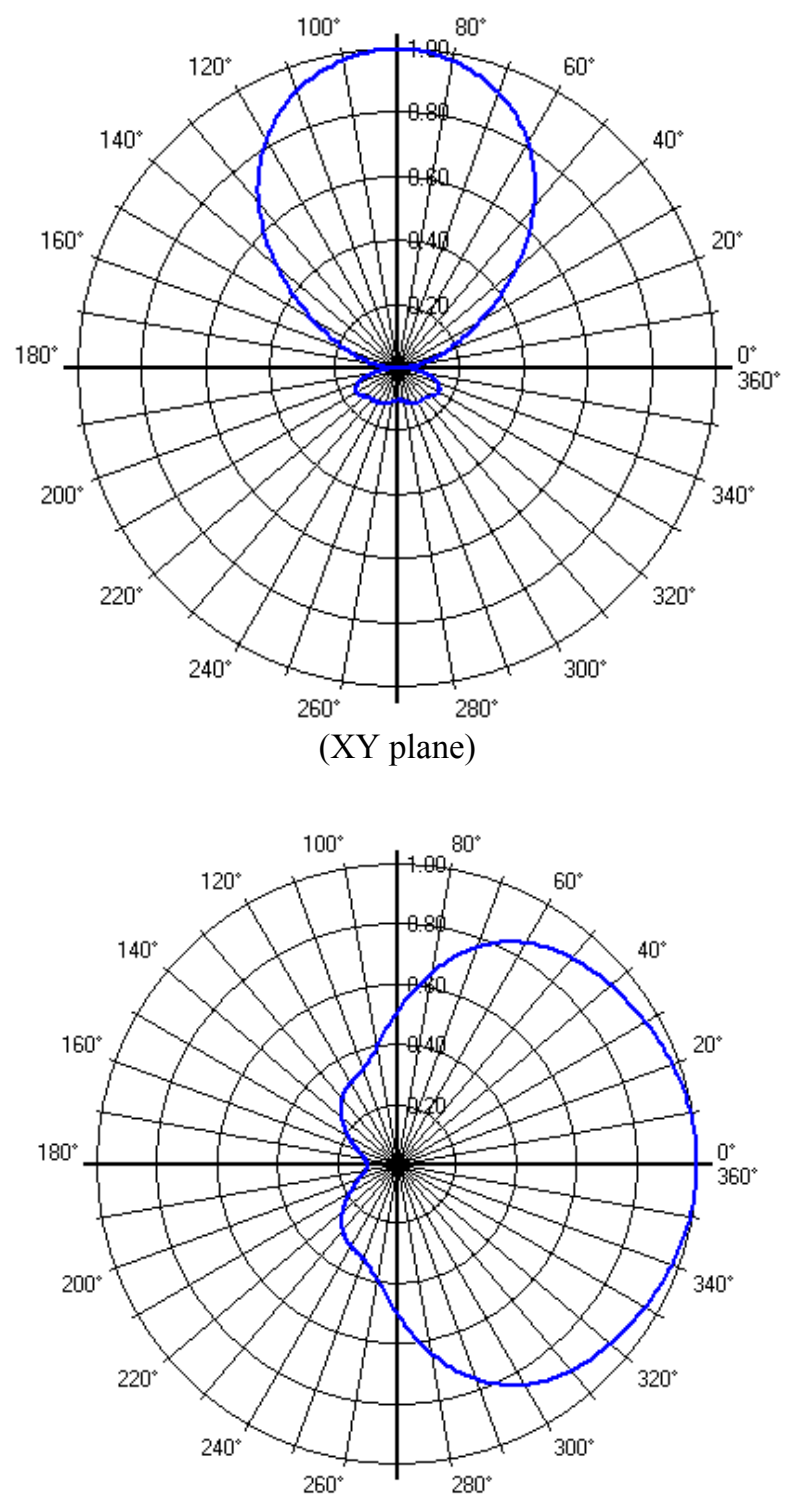

(YZ plane)

Figure 5: $\quad$ Radiation pattern at $f=250 \mathrm{MHz}$.

\section{Concluding remarks}

The frequency domain analysis of the log-periodic dipole array (LPDA) with boundary elements is undertaken in this work. The formulation is based on the 
set of coupled integro-differential equations of the Pocklington type. The induced currents along the elements of the array are obtained as the BEM solution of the Pocklington equations. Once obtaining the current distribution along the elements of LPDA the related far field patterns are computed.
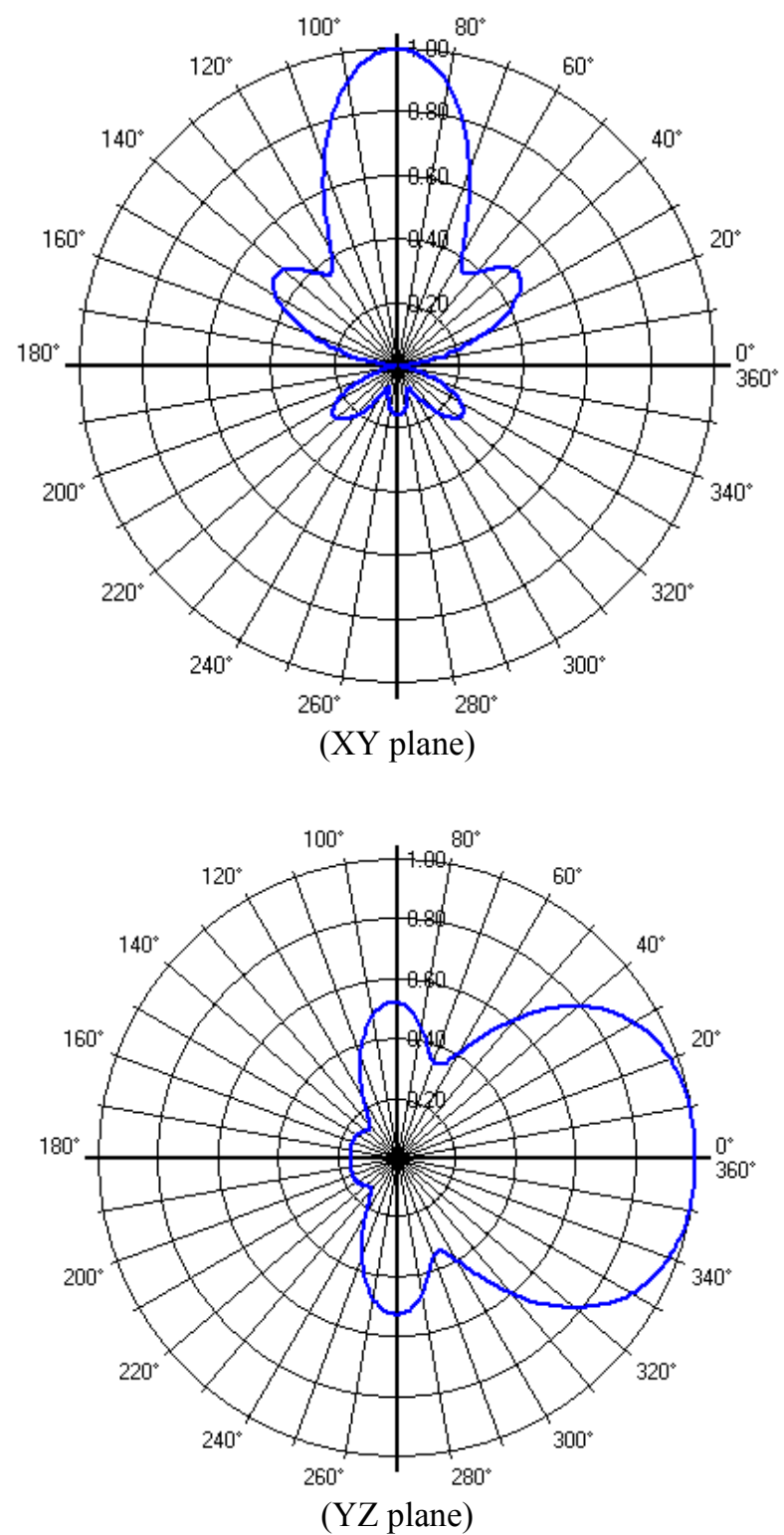

Figure 6: $\quad$ Radiation pattern at $f=300 \mathrm{MHz}$. (YZ plane). 


\section{References}

[1] R.A. Burberry, VHF and UHF Antennas, IEE Electromagnetics Series 35, Peter Peregrinus Ltd, London, UK, 1992.

[2] C.A. Balanis: Antenna Theory, 2nd Edition, John Wiley \& Sons, Inc., New York, 1997.

[3] Poljak, D., Doric, V., Roje, V., Galerkin-Bubnov Boundary element analysis of the Yagi-Uda array, 24th International Conference on the Boundary Element Method, BEM XXIV, pp 457-463, Sintra, Portugal, June 2002.

[4] Poljak D., C.A. Brebbia, Boundary Element Methods for Electrical Engineers, WIT Press, Southampton-Boston, 2005.

[5] Miller, E.K., Poggio, A.J., Burke, G.J., Selden, E.S., Analysis of wire antennas in the presence of a conducting half-space. Part II. The horizontal antenna in free space, Canadian Journal of Physics, 50, 1972, pp 26142627.

[6] Sarkar, T.K., Analysis of arbitrarily oriented thin wire antennas over a plane imperfect ground, Archiv fur elektronik und ubertragungstechnik, 31, 1977, pp 449-457.

[7] Parhami, P. Mittra, R., Wire antennas over a lossy half-space, IEEE Trans. $A P, \mathbf{2 8}, 1980$, pp 397-403.

[8] D.Poljak, V.Doric, S.Antonijevic "Computer aided Design of Wire Structures: Frequency and Time Domain Analysis", WIT Press, Southampton-Boston, 2007. 